\title{
Reinstating object-based attention under positional certainty: The importance of subjective parsing
}

\author{
ZHE CHEN \\ University of Canterbury, Christchurch, New Zealand \\ and \\ KYLE R. CAVE \\ University of Massachusetts, Amherst, Massachusetts
}

\begin{abstract}
Previous studies have shown that interference from flanking distractors can be modulated by the object organization of the scene. The experiments reported here test for object-based attention under conditions of positional certainty, which allow a narrow focus of attention to the target. Prior research has suggested that object-based attention does not arise in these circumstances, but the experiments presented here show that object-based attention can still appear if previous experience with the stimuli leads participants to interpret the stimulus pattern as two separate objects. Two control experiments demonstrate that the appearance of object-based attention is not due simply to a widening of the focus of spatial attention. The presence of object-based attention in such a focused-attention task argues against Shomstein and Yantis's (2002) proposed explanation of object-based attention based on priority in the order of visual search.
\end{abstract}

A number of different experimental paradigms have demonstrated that the object organization of a scene can affect the allocation of attention, even when object organization is irrelevant to the task. One paradigm, which originated with Egly, Driver, and Rafal (1994), comprised two simple rectangles and a spatial cue that appeared at the end of one of the rectangles. Responses to a test stimulus at the cued location were faster than responses to a test stimulus at the uncued end of the same rectangle, confirming earlier studies showing that attention weakens with distance from the cue (Downing \& Pinker, 1985; LaBerge \& Brown, 1989). However, test stimuli at the uncued end of the cued rectangle still elicited faster responses than stimuli on the other rectangle, which was completely uncued. Attention favored all parts of the cued object to some extent.

Other experiments using other techniques have demonstrated that this object-based attention extends to groups of objects that share a basic feature such as color. Harms and Bundesen (1983) used inhibition from flanking distractors to show that a distractor that was the same color as the target was to some extent selected with the target, even though the distractor was irrelevant to the task and

This research was supported by Grant U6498 from the University of Canterbury to Z.C., and in part by a University of Canterbury Visiting Erskine Fellowship to K.R.C. We thank Catherine Arrington, Yuhong Jiang, Sarah Shomstein, and an anonymous reviewer for their helpful comments. Correspondence should be addressed to Z. Chen, Department of Psychology, University of Canterbury, Private Bag 4800, Christchurch, New Zealand (e-mail: zhe.chen@canterbury.ac.nz). the location of the target was known before it appeared. Kim and Cave (2001) used spatial probes to show that the target-colored distractor also received more spatial attention than a distractor of a different color. Similar evidence has been provided by Kramer and Jacobson (1991).

However, as Shomstein and Yantis (2002) demonstrated, the effects of object organization do not always appear. In four of their five experiments, the location of the target letter to be reported was known in advance, allowing attention to be focused at that location in preparation for the stimulus. Flanking distractor letters were also present in the displays, sometimes within the same rectangle as the target and sometimes within a different rectangle. As expected, the flanking distractors interfered with the response when they were near the target (B. A. Eriksen \& C. W. Eriksen, 1974), but the amount of interference was no greater when the distractors were within the same rectangle as the target than when they were in different rectangles. In the fifth experiment, the location of the target varied from trial to trial. This time, the object effect appeared, with more distractor interference when targets and distractors were within the same rectangle.

Shomstein and Yantis's (2002) results suggest that object effects appear only with spatial uncertainty. In their study, the object boundaries affected performance only when the target location was not known, and attention thus could not be focused at a single location. However, object-based effects do appear in the experiments by Harms and Bundesen (1983), Kim and Cave (2001), and Kramer and Jacobson (1991), in all of which the target location was known. With this apparent conflict between the different results, it is not 
clear whether the deployment of object-based selection depends on spatial uncertainty or on other factors.

Knowing the link between spatial uncertainty and objectbased attention is important for understanding how object-based attention arises within visual processing. Object-based attention is generally explained either in terms of selecting abstract, location-independent representations (Vecera \& Farah, 1994) or in terms of selecting a region of the visual field belonging to the object without selecting other regions (Kim \& Cave, 1995, 2001; Kramer, Weber, \& Watson, 1997). In both accounts, attention is assumed to improve the quality of the perceptual representation of the selected item or its region. Shomstein and Yantis (2002), however, interpreted their results to mean that object-based attention occurs through the setting of priorities for search. According to their account, the priority that different parts of a scene receive during the guidance of visual search depends partly on the object organization of the scene. This guidance is unnecessary when the target location is known in advance, and thus, with positional certainty and a narrow focus of attention, there is no opportunity for object effects. Therefore, answering the question about the relationship between spatial uncertainty and object-based effects will help to determine whether object-based attention arises from the activation of visual representations or is simply the by-product of guidance in search.

In the experiments presented here, we investigate the link between spatial certainty and object-based attention. In all of the experiments, the target letter to be reported always appeared in the center of the display, and its location was known in advance. In Experiment 1, we used methods similar to those used by Shomstein and Yantis (2002) in that the three rectangles that made up the stimulus pattern were presented together on every trial. The results replicated the findings of Shomstein and Yantis: The amount of interference from the flanking distractors was unaffected by the object organization of the stimulus. In Experiment 2, we introduced filler trials in which just one part of the stimulus array appeared. The results showed that the presence of the fillers was sufficient to produce an object effect, presumably because previous experience with the shape components made it more likely that they would be perceived as separate objects. Thus, spatial certainty by itself cannot always eliminate an object effect. Experiments 3 and 4 included spatial probes to measure the allocation of spatial attention as the task was performed, in order to rule out an alternative explanation and to provide evidence as to how object-based attention was accomplished in the task.

\section{EXPERIMENT 1}

The goal of Experiment 1 was to replicate the results of Shomstein and Yantis (2002) using a paradigm similar to theirs. As in their experiments, the target display consisted of three rectangles (a large one and a pair of smaller ones), and the target always occurred at the center of the large rectangle. The flankers, which were either unrelated to or incompatible with the target response, could occur on the same rectangle as the target or on different rectangles. On the basis of Shomstein and Yantis's results, it was predicted that no object-based modulation of flanker interference would be found.

\section{Method}

Participants. Sixteen undergraduate students from the University of Canterbury took part in the experiment for payment. All reported having normal or corrected-to-normal vision.

Apparatus and Stimuli. Stimulus displays were shown on a 13-in. RGB monitor of a Power Macintosh 6100/66 computer. The participants viewed the monitor from a distance of approximately $60 \mathrm{~cm}$ in a dim room. MacProbe (1.6.9) was used to display stimuli and to record responses.

All displays were presented against a homogeneous gray background. The stimulus display consisted of one large and two small rectangles outlined in blue (see Figure 1). For half of the trials, the large rectangle was oriented horizontally and the small rectangles were oriented vertically, and for the other half the orientations were reversed. At a viewing distance of approximately $60 \mathrm{~cm}$, the large rectangle subtended $9.55^{\circ} \times 1.24^{\circ}$, and the two small ones each subtended $4.06^{\circ} \times 1.24^{\circ}$. The small rectangles were placed on either side of the large rectangle, with a gap subtending a visual angle of $0.095^{\circ}$. Thus the entire display subtended $9.55^{\circ}$ in both length and width.

The stimulus pattern was displayed first, and it remained on the screen as three black letters appeared simultaneously. Each letter was an uppercase $\mathrm{H}, \mathrm{S}$, or $\mathrm{O}$ in 30-point Geneva font. The letters were aligned either horizontally or vertically. Depending on the specific experimental condition on a given trial, the letters would either all appear on the large rectangle, or the target letter would appear on the large rectangle and the two flankers would appear on the small rectangles. In all cases, the gap between any two adjacent letters subtended $0.57^{\circ}$.

Design and Procedure. The experiment employed a repeated measures design. The task was to decide whether the center letter was an $\mathrm{H}$ or an S. The principal manipulations were the relative position of the target and the flankers (i.e., whether they were on the same object or on different objects) and their compatibility (whether the flankers were neutral or incompatible with respect to the target response). The target and flankers were equally likely to be on the same object as on different objects, and the number of trials for which the target and flankers were unrelated was equal to the number of trials for which they were associated with different responses. Altogether, there were four conditions: same-neutral (SN), on which the target and flankers were on the same object and the flankers were not associated with any responses; same-incompatible (SI), on which the target and flankers were on the same object but required different responses; different-neutral (DN), on which the target and flankers were on different objects and the flankers were not associated with any responses; and differentincompatible (DI), on which the target and flankers were on different objects and required different responses.

Each trial began with the display of three rectangles, which remained visible throughout the trial. After $1,125 \mathrm{msec}$, a target and two flanking letters were flashed on screen for $120 \mathrm{msec}$. The target, which was the middle letter, could be an $\mathrm{H}$ or an S. In the incompatible condition, it was flanked on the left and the right or on the top and the bottom by the other member of the target set; in the neutral condition, by the letter $\mathrm{O}$. No feedback was provided during the experiment, and the intertrial interval was $1,500 \mathrm{msec}$.

The participants were told that they would see two sets of rectangles, a large one and a pair of small ones, and that the rectangles would be presented together to form a pattern, with three letters displayed either horizontally or vertically. Their task was to respond to the middle letter on each trial by pressing the " $<$ " key for S and the " $>$ " key for $\mathrm{H}$ with the index or middle finger of their dominant hand. Both speed and accuracy were emphasized. After 32 practice 

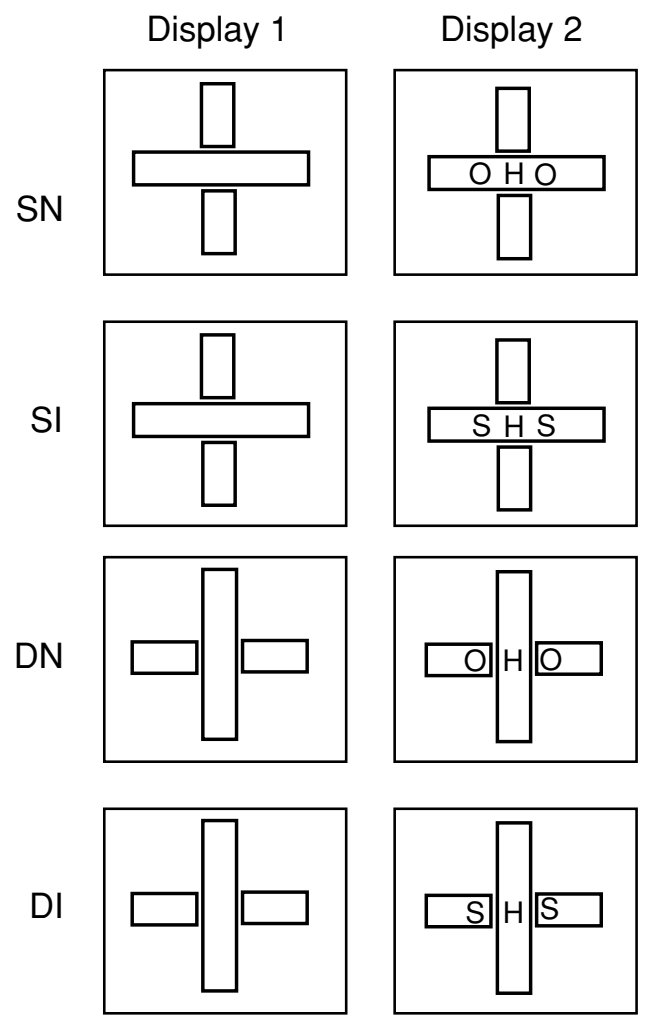

Figure 1. Examples of stimulus displays from Experiment 1. Each trial started with the presentation of a stimulus pattern followed by a target display. The task was to decide whether the central letter was an $\mathrm{H}$ or an $\mathrm{S}$. On half of the trials in each condition, the configuration of rectangles was oriented so that the large rectangle was horizontal, and on the other half the large rectangle was vertically oriented. SN refers to the same-neutral condition, in which the target and flankers were on the same object and the flankers were not associated with any responses; SI refers to the same-incompatible condition, in which the target and flankers were on the same object but they indicated different responses; DN refers to the different-neutral condition, in which the target and flankers were on different objects and the flankers were unrelated to any responses; and DI refers to the differentincompatible condition, in which the target and flankers were on different objects and they indicated different responses.

trials, each participant performed 4 blocks of 160 trials. The experiment took about $35 \mathrm{~min}$ to complete.

\section{Results and Discussion}

The mean reaction times and accuracy data for 15 participants are shown in Table 1..$^{1}$ One participant did not finish the experiment due to a fire drill. A repeated measures ANOVA on reaction times revealed a main effect of flanker position $[F(1,14)=28.41, p<.001]$ as well as a main effect of compatibility $[F(1,14)=50.70, p<$ $.001]$. Responses were faster when the target and flankers were on different objects $(455 \mathrm{msec})$ than when they were on the same object $(466 \mathrm{msec})$. Reaction times were also faster when the flankers were neutral $(450 \mathrm{msec})$ than when they were incompatible $(470 \mathrm{msec})$. The two-way interaction between position and compatibility was not significant $[F(1,14)=0.48$, n.s. $]$.

A similar analysis was conducted on the accuracy data. A main effect of compatibility was found $[F(1,14)=$ $6.86, p<.05]$, suggesting higher accuracy when the flankers were neutral $(2.2 \%$ error) than when they were incompatible (3.5\% error). No other effects approached significance, and there was no indication of a speedaccuracy trade-off.

As the above analyses show, our results bear remarkable similarities to the findings of Shomstein and Yantis (2002, Experiments 1-4). Like the participants in their experiments, the participants in our study showed a response compatibility effect, suggesting that the presence of irrelevant information in close proximity to the target impaired their ability to process it efficiently. A similar flanker interference effect has been reported in many prior studies, and is known to be a robust phenomenon (B. A. Eriksen \& C. W. Eriksen, 1974; C. W. Eriksen \& St. James, 1986). In addition, the participants also demonstrated a flanker position effect in that they were faster to respond to the target when it appeared on a different object from the flankers than when it appeared on the same object. Shomstein and Yantis reported a similar effect in their Experiment 1, and attributed this effect to the result of a segmentation process that participants evoked to separate the target from the flankers when both occurred on the same object.

The most important result with respect to the purpose of the present experiment is the absence of an object-based modulation of flanker interference. In other words, the degree of flanker interference was similar regardless of whether the target and flankers were on the same object or on different objects. This result replicates the finding of Shomstein and Yantis (2002) and is consistent with their priority account of object effects. Because the location of the target was invariant in our experiment, there was no apparent reason for the participants to prioritize their search to any location other than the target location itself. If object effects are the result of an object-specific attentional prioritization strategy, as Shomstein and Yantis proposed, it is not surprising that no differential levels of flanker interference were observed, since such a strategy was not necessary.

Table 1

Mean Reaction Times (in Milliseconds) and Error Rates (Percentage Incorrect), With Standard Errors, for Experiment 1

\begin{tabular}{lrrrrr}
\hline & \multicolumn{2}{c}{ RT } & & \multicolumn{2}{c}{$\%$ Error } \\
\cline { 2 - 3 } \cline { 5 - 6 } & $M$ & $S E$ & & $M$ & $S E$ \\
\hline Same Object & & & & \\
I & 476 & 12.6 & & 4.0 & 1.00 \\
N & 455 & 12.6 & & 2.0 & 0.43 \\
I - N & 21 & 3.1 & 2.0 & 0.84 \\
Different Object & & & & \\
I & 464 & 12.5 & 3.0 & 0.85 \\
N & 445 & 12.5 & 2.4 & 0.64 \\
I - N & 19 & 4.3 & 0.6 & 0.41 \\
\hline
\end{tabular}

Note-I, incompatible; N, neutral. 
However, a close inspection of the experiment suggests an alternative interpretation. Recall that in this experiment as well as in those of Shomstein and Yantis (2002), the three rectangles that comprised the stimulus pattern were always presented together. Although it was emphasized to the participants in the instructions that the stimulus pattern was made of two sets of rectangles, there was nothing in the pattern to encourage them to parse the image in that way. When attention was focused narrowly at the center, the fact that there were two different types of stimulus configuration - one with the large rectangle on the horizontal axis and the other with it on the vertical axis - might have become less salient over time. This in turn would have discouraged the perception of the stimulus pattern as a configuration of three rectangles rather than as a single entity such as a cross. It is possible that it was perceived in that way on at least some of the trials.

Past research has shown that the manifestation of object effects is influenced by a number of factors, including the quality of the object representation and the participants' subjective organization of a stimulus pattern (Avrahami, 1999; Chen, 1998; Watson \& Kramer, 1999). For example, response latencies to a target differ as a function of whether the stimulus pattern is perceived as being made of one object or two objects (Chen, 1998). Specifically, when the stimulus configuration was described as two colored $V_{\mathrm{S}}$ that were partly superimposed at the base, the participants showed an object effect: They were faster to switch attention between the two arms of the same $V$ rather than between the two different $V_{\mathrm{S}}$. In contrast, when the same physical stimulus was described as an $X$ made of two different colors, the previously observed object effect disappeared. It is important to note that both experiments included filler trials, displays that consisted of a single color $V$ (for the $V$ experiment) or a single color $X$ (for the $X$ experiment) to induce the participants to perceive the bicolored stimulus configuration as either a $V$ or an $X$.

With respect to the present experiment, if the subjective parsing of the stimulus pattern contributed to the lack of an object effect, then inducing participants to see the stimulus pattern as being made of separate objects would facilitate object-based distribution of attention, which in turn would lead to a differential magnitude of flanker interference between same and different object conditions. Experiment 2 tested this hypothesis.

\section{EXPERIMENT 2}

Experiment 2 was the same as Experiment 1 except that the three rectangles were presented together on only half the trials (the experimental trials). On the rest of the trials, either only the large rectangle or only the pair of small ones was shown. These were filler trials whose function was to induce participants to see the stimulus pattern on the experimental trials as being made of two sets of distinct objects. Of particular interest was whether the inclusion of the filler trials would result in object-based distribution of attention.

\section{Method}

Trials in Experiment 2 were identical to those in Experiment 1, except that half of them were replaced by filler trials consisting of equal numbers of either the large rectangle or the two small ones. The orientation of the stimulus was horizontal on half of the filler trials and vertical on the rest. On trials with the single large rectangle, the target was displayed in the center of the rectangle. On trials with the two small rectangles, the target was presented directly against the background between the rectangles (see Appendix A). Fifteen new paid participants took part in the experiment.

\section{Results and Discussion}

Table 2 illustrates the mean reaction time and accuracy data for the experimental trials of Experiment 2. (The data for the filler trials are shown in Appendix B.) Analyses similar to those in Experiment 1 were conducted. For the reaction times data, all of the effects were significant. As in Experiment 1, reaction times were shorter when the target and flankers were on different objects $(430 \mathrm{msec})$ than when they were on the same object $(437 \mathrm{msec})[F(1,14)=$ $9.54, p<.01]$. They were also shorter when the flankers were neutral $(422 \mathrm{msec})$ rather than incompatible $(445 \mathrm{msec})[F(1,14)=43.01, p<.001]$. Furthermore, the flanker compatibility effect was larger when the target and distractors were on the same object $(32 \mathrm{msec})$ than when they were on different objects $(15 \mathrm{msec})[F(1,14)=$ $18.17, p<.001]$.

Regarding accuracy, the participants were more accurate when the flankers were neutral than when they were incompatible: $2.4 \%$ versus $4.5 \%$ error for the neutral and incompatible trials, respectively $[F(1,14)=9.27, p<$ $.01]$. No other effects reached significance.

The most important finding of Experiment 2 is the observation of object-based modulation of flanker interference. The participants showed greater interference from incompatible flankers when the flankers were on the same object as the target than when they were on different objects from the target. Because the major difference between Experiments 1 and 2 was the presence of the filler trials in Experiment 2, it seems reasonable to attribute the different results of Experiment 2 to the inclusion of filler trials. To confirm that the participants showed different patterns of response as a function of the presence or absence of the filler trials, a combined analysis was performed across

Table 2

Mean Reaction Times (in Milliseconds) and Error Rates (Percentage Incorrect), With Standard Errors, for Experiment 2

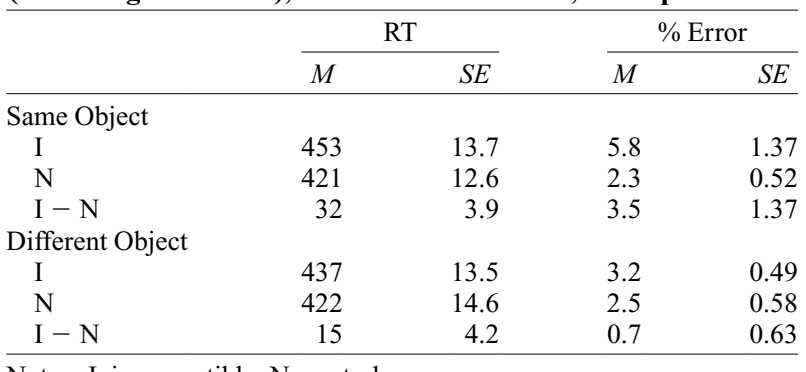

Note-I, incompatible; N, neutral. 
the two experiments. A mixed ANOVA on reaction times revealed that in addition to the significant main effects of flanker position $[F(1,28)=33.00, p<.001]$, compatibility $[F(1,28)=91.37, p<.001]$, and position $\times$ compatibility interaction $[F(1,28)=10.64, p<.01]$, there was also a significant three-way interaction among experiment, flanker position, and compatibility $[F(1,28)=4.83$, $p<.05]$. This last effect suggests that the participants in Experiments 1 and 2 behaved differently with respect to object-based distribution of attention.

One might argue that the difference in results between Experiments 1 and 2 could be attributed to the different number of experimental trials in the two experiments rather than to the absence or presence of the filler trials. Because there were an equal number of trials in the two experiments, once the filler trials were excluded from the data analyses, the results of Experiment 2 were in fact based on half the number of trials as the results in Experiment 1 . If the manifestation of the object effect is sensitive to the quantity of stimulus exposure, the difference in the number of experimental trials in the two experiments could lead to their different results. There is some evidence in prior research that the quantity of stimulus exposure affects subjective organization. Yantis (1992) reported that participants performed better in tracking randomly moving dots when they were instructed to group the dots as a higher order "object." However, the effect dissipated in the latter part of the experiment. This suggests that grouping by subjective organization can be extremely sensitive, especially when there are equally plausible ways to perceive the structure of a stimulus pattern.

To examine whether object-based attentional distribution changed over time in Experiment 1, the first and the second halves of participants' data were divided and analyzed separately. Repeated measures ANOVAs with part (first vs. second), flanker position, and compatibility as within-subjects variables were conducted on reaction time and accuracy. No significant interactions involving part were found, suggesting that the object-based modulation of flanker interference was comparable in the first and the second halves of Experiment 1. Thus, it is unlikely that the difference in the results of Experiments 1 and 2 was caused by the different number of experimental trials between the two experiments.

If we assume that the filler trials played a crucial role in the manifestation of the object effect in Experiment 2, the question arises as to why object effects were observed in many other experiments in which filler trials were not employed. Although it is difficult to identify the exact cause because of the many differences in methodology, an important factor may be the structure of a stimulus pattern, which is known to influence processing strategies (Garner, 1970, 1974; Garner \& Felfoldy, 1970; Gottwald \& Garner, 1975). A careful inspection of the various stimulus patterns in experiments on object-based attention revealed an interesting feature. In the studies in which an object effect was not found (Experiments 2A \& 2B of Chen, 1998, and Experiments 1-4 of Shomstein \& Yantis, 2002), the stimulus patterns tended to resemble a single object (e.g., an X or a cross). In contrast, in the majority of other studies where an object effect was reported (Duncan, 1984; Egly et al., 1994), the stimulus patterns looked more like two separate objects (e.g., a bar on top of a rectangle or two bars).

To verify whether the filler trials in Experiment 2 could affect the participants' spontaneous parsing of the stimulus pattern, we conducted a survey of 16 randomly selected students. Each was shown a sequence of four stimulus displays. Half of the participants saw only the whole configurations (the whole group) whereas the rest of them saw two whole and two part configurations (the mixed group). The last stimulus in the sequence was always a whole one. The participants were instructed to describe the last stimulus with the first thing that came to their mind. Interestingly, 7 of 8 participants in the whole group replied with the word "cross." In contrast, only one participant in the mixed group gave the same answer. The rest of the group provided a variety of responses including "children's playing blocks," "line on the row," "mathematical symbol," and "two smaller rectangles and one long one." These results are consistent with the notion that the presence or absence of the filler trials induced the participants to perceive the stimulus pattern differently. ${ }^{2}$

However, although our survey data provide clear evidence in support of the subjective parsing account of the previous experiments, it would be beneficial to examine one other alternative interpretation: the possibility that the filler trials induced a more relaxed attentional focus in Experiment 2. ${ }^{3}$ The extent of attentional focus is a known moderator of object-based attention (Atchley \& Kramer, 2001; Goldsmith \& Yeari, 2003). Goldsmith \& Yeari demonstrated in a series of elegant experiments that in spatial cuing tasks, object effects would emerge only if attention was spread diffusely either prior to or during the attentional deployment. In the present experiment, because the stimulus configuration on the filler trials was less complex than the one on the experimental trials, it may have induced the participants to focus attention less narrowly at the center. The random presentation of the two types of trials might have further encouraged the participants to use a broad attentional focus throughout the experiment. Thus, the differential degrees of object effects in Experiments 1 and 2 may have been due to the extent of attentional focus rather than to the subjective parsing of the stimulus pattern.

The possibility that the filler trials trigger object-based attention by generally widening the focus of spatial attention was examined in Experiments 3 and 4, in which spatial probes were used to measure how spatial attention was allocated as the task was performed. The response times from the probes may also provide some insight into how object-based attention is implemented in this type of task. As mentioned in the introduction, there are two different ways in which objects can be attended. On the one hand, object-based attention can be due to the selection of abstract, location-independent representations (Vecera \& Farah, 1994). This type of "pure" object-based selection operates separately from location-based attention; if the probe responses reflect the allocation of location-based attention, then we would expect no differences in responses 
to probes between conditions with and conditions without object-based selection. In other words, the pattern of responses to the spatial probes should be the same whether the filler trials are present or not.

On the other hand, object-based selection may be mediated by location selection, so that an object is selected by determining the spatial region it occupies and selecting that region (Kim \& Cave, 1995, 2001; Kramer, Weber, $\&$ Watson, 1997). With this sort of location-mediated object-based attention, the presence of the filler trials would cause attention to spread within the entire spatial region occupied by the rectangle that contained the target. Under this account, response times to probes should be shorter when the probes are within the same rectangle as the target but unchanged or longer when the probes are inside the other rectangles or outside the rectangles.

The third possible outcome is predicted by the alternative explanation that filler trials generally widen the focus of spatial attention. According to this account, adding the filler trials should produce faster responses to all probe locations away from the center, whether they are inside or outside the rectangles. The filler trials might also cause responses for probes at the center to be slower, or those responses might be no different from the condition without the filler trials.

Experiment 3 tested this alternative hypothesis by probing at the center and at four peripheral locations outside the rectangles. Peripheral locations within the rectangles were probed in Experiment 4.

\section{EXPERIMENT 3}

In Experiment 3, we employed a spatial probe paradigm to measure how the spread of attention was affected by the presence or absence of filler trials. In a typical probe experiment (Cave \& Zimmerman, 1997; Kim \& Cave, 1995, 1999), participants perform a primary task such as searching for a specific target among distractors. They do not give their response to the search until prompted for it by the computer. Thus, there is a delay between the offset of the search display and the onset of the prompt. On some trials, a probe stimulus appears at one of several locations during the delay period, and the participants are required to press a key as soon as the probe occurs. Detection of the probe is generally faster when it appears at a location previously occupied by a target rather than by a distractor (Kim \& Cave, 1995). These results suggest that reaction time to the probe is sensitive to the allocation of spatial attention. The probe paradigm can be seen as an extension of the standard attentional cuing paradigm (C. W. Eriksen \& Hoffman, 1974; Posner, Snyder, \& Davidson, 1980), with attention being directed by a primary task rather than by a spatial cue.

We incorporated the probe technique into our existing paradigm, and tested two groups of participants. All participants saw stimulus displays similar to those used in Experiments 1 and 2, with a spatial probe added on some of the trials. The probe was a small dot, and it could ap- pear either at a central location or at one of four peripheral locations outside of the three rectangles. The filler trials were intermixed with the experimental trials for the mixed group; they were excluded from the experimental trials for the whole group. If the presence of the filler trials induced a more relaxed attentional focus in Experiment 2, the participants in the mixed group of the present experiment would adopt a broader attentional focus than those in the whole group. This in turn would result in different patterns of reaction times across central and peripheral probes between the two groups. Conversely, if there were no differences in the patterns of probe responses across the two groups, then it is unlikely that there was a difference in the extent of attentional focus between Experiments 1 and 2 .

\section{Method}

Figure 2 shows the sequence of displays in Experiment 3. As in the previous experiments, each trial started with the display of a stimulus pattern. After $1,125 \mathrm{msec}$, a target and two flankers were presented, and they remained on the screen for $120 \mathrm{msec}$. To allow accurate measurement of response time to the probes that appeared after the offset of the letters on some of the trials, the participants were required to wait before giving their response to the letterreporting task until a prompt appeared. On the no-probe trials, which comprised three-fourths of the total number of trials, the prompt was presented $1,035 \mathrm{msec}$ after the offset of the letters. On the remaining trials, a probe consisting of a small white dot subtending $0.19^{\circ}$ appeared $30 \mathrm{msec}$ after the offset of the letters and remained on the screen for $60 \mathrm{msec}$. The probe could appear at the target location in the center or at one of four peripheral background locations $4.78^{\circ}$ away from the center between the arms of the cross. (The probe locations were identical on the filler trials.) The participants used their left index finger to press the " $Z$ " key as soon as the probe appeared. Upon their response, the prompt for the target letter was displayed. The participants used their right index finger to respond to the target letter, and accuracy was stressed for the letter task. Whereas both reaction time and accuracy were measured for the probe trials, only accuracy was recorded for the letter task. For one group of participants, all of the trials were experimental ones, with all three rectangles appearing. For the other group, half of the trials were filler trials. All other aspects of the experiment were identical for the two groups. Sixteen new paid participants volunteered for the experiment.

\section{Results and Discussion}

The results are shown in Tables $3 \mathrm{~A}$ and $3 \mathrm{~B}$ (see Appendix $\mathrm{C}$ for the filler trials). For the probe trials, mixed ANOVAs were conducted on both the accuracy and reaction time data. The only significant result was the main effect of location on reaction time $[F(1,14)=16.07, p<$ $.01]$. The participants were faster to detect the probe when it occurred at a peripheral location (443 $\mathrm{msec})$ than when it occurred at the center $(469 \mathrm{msec})$. This result was presumably caused by the masking effect of the target, which appeared at the same location before the onset of the central probe. Because there was nothing to precede the appearance of the probes at a peripheral location, they were detected faster than a central probe.

For the letter task, a mixed ANOVA showed that the error rates were higher when a probe followed the presentation of the letters than when there was no probe on that trial: $2.43 \%$ error versus $0.49 \%$ error for the probe and 


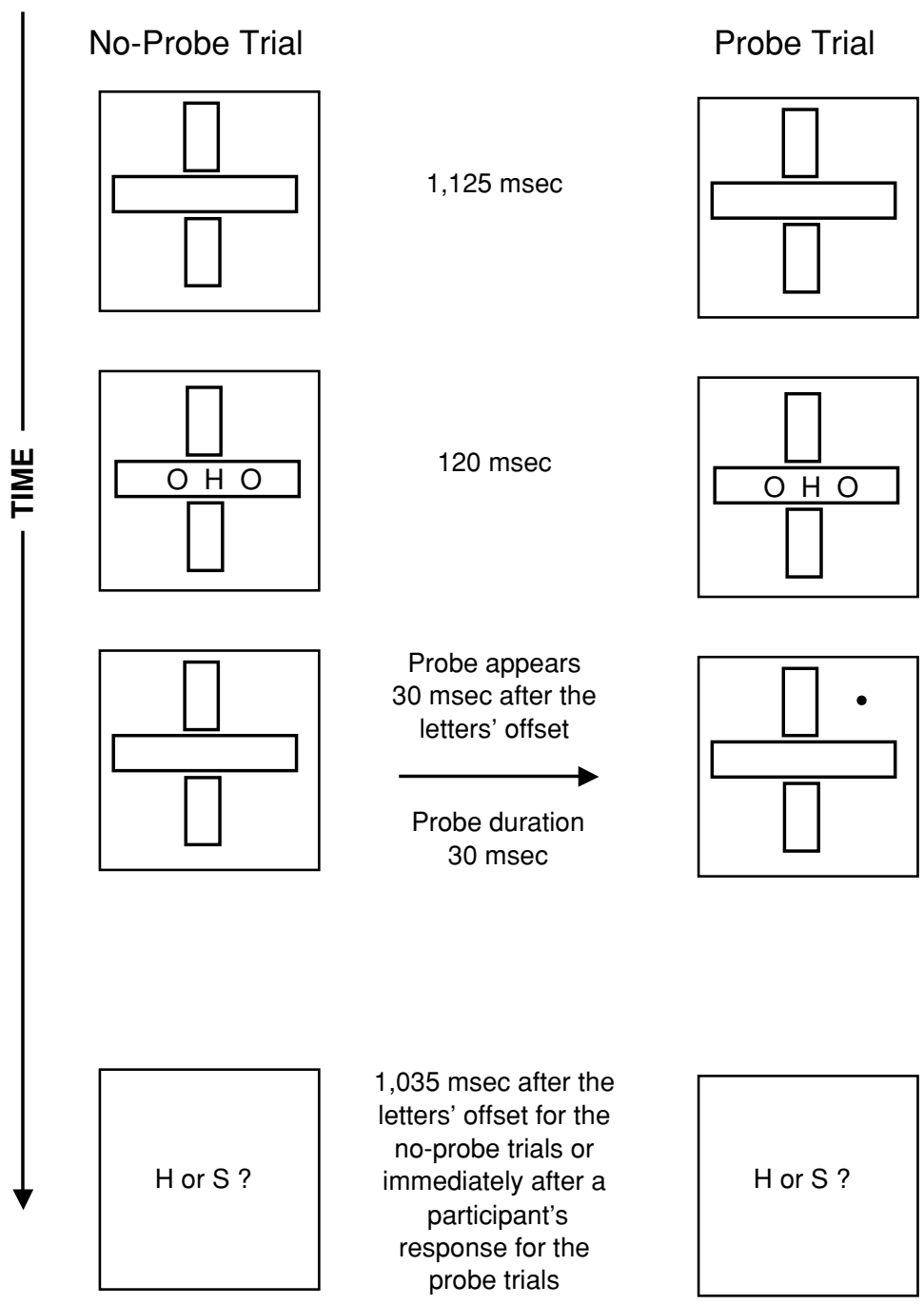

Figure 2. Examples of stimulus displays from Experiment 3. Each trial started with the presentation of a stimulus pattern, followed by a letter display. Participants held their response to the target letter until a prompt appeared at the end of a trial. On three fourths of the trials (the no-probe trials), the prompt occurred $1,035 \mathrm{msec}$ after the offset of the letters. On the remaining trials, a probe appeared at either the target location in the center or at one of four peripheral background locations. The task was to respond to the probe as quickly as possible. Upon response, the prompt for the target letter was displayed. Participants were divided into two groups. Depending on the specific group, they either saw only the experimental trials, or both the experimental and the filler trials.

no-probe trials, respectively $[F(1,14)=25.68, p<.001]$. This result suggests that the onset of the probe and/or its response impaired the participants' ability to perform the letter task. It should be noted, however, that the overall accuracy remained very high even on the probe trials, suggesting that the impairment was limited. No other effects reached significance.

The absence of any group differences between the patterns of data across central and peripheral probes makes it unlikely that the participants in Experiments 1 and 2 differed in the extent of their attentional focus on the ex- perimental trials. Although the results of Experiment 3 in isolation do not allow us to draw definitive conclusions regarding the spread of attention in the previous experiments, the overall pattern of results from the three experiments suggests that the differential degrees of object effect observed in Experiments 1 and 2 were caused by subjective parsing of the stimulus pattern rather than by the differential extents of attentional focus.

However, before any definitive conclusion can be drawn, we need to consider one other possibility: the allocation of attention may be different inside the object 
Table 3A

Mean Reaction Times (in Milliseconds) and Error Rates (Percentage Incorrect), With Standard Errors, for the Probe Detection Task in Experiment 3

\begin{tabular}{llllll}
\hline & \multicolumn{2}{c}{ RT } & & \multicolumn{2}{c}{$\%$ Error } \\
\cline { 2 - 3 } \cline { 5 - 6 } & $M$ & $S E$ & & $M$ & $S E$ \\
\hline Whole Group & & & & \\
$\quad$ Central & 468 & 20.4 & & 0.0 & 0.0 \\
$\quad$ Peripheral & 444 & 19.9 & & 1.27 & 0.39 \\
Mixed Group & & & & \\
$\quad$ Central & 470 & 20.7 & & 3.13 & 1.67 \\
$\quad$ Peripheral & 441 & 30.1 & & 1.37 & 0.46 \\
\hline
\end{tabular}

Table 3B

Mean Error Rates (Percentage Incorrect), With Standard Errors, for the Letter Discrimination Task in Experiment 3

\begin{tabular}{lccccc}
\hline & \multicolumn{2}{c}{ Whole Group } & & \multicolumn{2}{c}{ Mixed Group } \\
\cline { 2 - 3 } \cline { 6 - 6 } & \% Error & $S E$ & & \% Error & $S E$ \\
\hline With probe & 1.72 & 0.39 & & 3.13 & 0.88 \\
Without probe & 0.18 & 0.05 & & 0.79 & 0.26 \\
\hline
\end{tabular}

boundaries, even though it was not appreciably different outside the object boundaries. Perhaps the filler trials in Experiment 3 facilitated the spread of attention within the boundaries of the objects on all of the trials in the mixed group. If the effect of the fillers was a general widening of attention that was confined to the region inside the object boundaries, it could be detected by positioning the peripheral probes inside rather than outside the rectangles. If filler trials triggered this more specific type of widening of spatial attention, then there should be generally faster responses to probes at peripheral locations within the rectangles in the filler trial condition.

If there was no general widening of spatial attention, then the peripheral probes within the rectangles would also allow us to compare the amount of attention allocated to the rectangles with targets and without. This comparison thus tests whether the object-based attention in this task is mediated by selection of the locations within the rectangles with targets.

\section{EXPERIMENT 4}

Experiment 4 examined the distribution of attention inside the stimulus configuration as a function of the presence or absence of the filler trials. As in Experiment 3, there were two groups of participants, and again the filler trials comprised half of the total trials for one group and were excluded from the experiment for the other group. Unlike Experiment 3, in which the spatial probe in the peripheral condition of the whole pattern was situated outside the object boundaries, the probe in the present experiment appeared inside one of the three rectangles. It could appear at a central location, at a peripheral location on the same rectangle as the target (the same condition), or at a peripheral location on a different rectangle from the target (the different condition). Two sets of comparisons were of particular interest. First, a comparison of the patterns of reaction times between the two groups across all probe locations would show the effect of the filler trials. If no significant difference was found between the two groups, this would provide converging evidence to the results of Experiment 3 that the presence of the filler trials did not generally widen the distribution of spatial attention. Second, a comparison between probes in peripheral locations in the same object as the target and those in a different object than the target would provide evidence as to whether the object-based attention in this task was accomplished by selecting all locations within the target object.

\section{Method}

The method was identical to that of Experiment 3 with two exceptions (see Figure 3). First, the probes always appeared inside the object boundaries, either at the center or near one of the outer ends of one of the three rectangles. In the latter case, the separation between the nearest point of the probe and the edge of the rectangle was $0.28^{\circ}$ degrees of visual angle. As before, the probe locations were identical on the filler trials. Second, to minimize the differences in masking among the four peripheral locations on the whole-pattern trials, two additional flankers were added, resulting in four identical flankers on each trial. Sixteen volunteers from the same participant pool took part in the experiment. Half of them were in the whole group, and the rest were in the mixed group. All other aspects of the experiment were identical to those of Experiment 3.

\section{Results and Discussion}

The results are shown in Tables 4A and 4B (see Appendix $\mathrm{D}$ for the results for the filler trials). For the probe trials, a mixed ANOVA on reaction time showed a main effect of location $[F(1,28)=24.26, p<.001]$. A Tukey HSD test further indicated that for the mixed group, the reaction time was faster in the same object condition $(398 \mathrm{msec})$ than in the central condition $(440 \mathrm{msec})$, and for the whole group, it was faster in both the same and the different object conditions $(410 \mathrm{msec}$ and $411 \mathrm{msec}$, respectively) than in the central condition $(452 \mathrm{msec})$. As in Experiment 3, the slower reaction times at the center were probably caused by the masking effect of the target. No other comparisons of pairs reached significance. Furthermore, neither the main effect for group $[F(1,14)=$ 0.1$, n.s. $]$ nor for group by location interaction $[F(2,28)=$ 0.69, n.s.] was significant. No reliable effects were found in accuracy, either.

For the letter task, the only significant effect was the main effect of probe. Error rates were higher when trials contained a probe than when there was no probe $[2.93 \%$ vs. $0.22 \%$ error, $F(1,14)=13.07, p<.01$ ], suggesting that the appearance of the probe and/or the response to it to some extent disrupted the perception or the maintenance of the target presentation.

The first question addressed by this experiment was whether the presence of the filler trials would cause a general spread of spatial attention. The answer to this question is clearly no. The distribution of attention across the different probed locations did not change with the addition of the filler trials. The data that would most directly indicate a spread of attention are the probe response times 


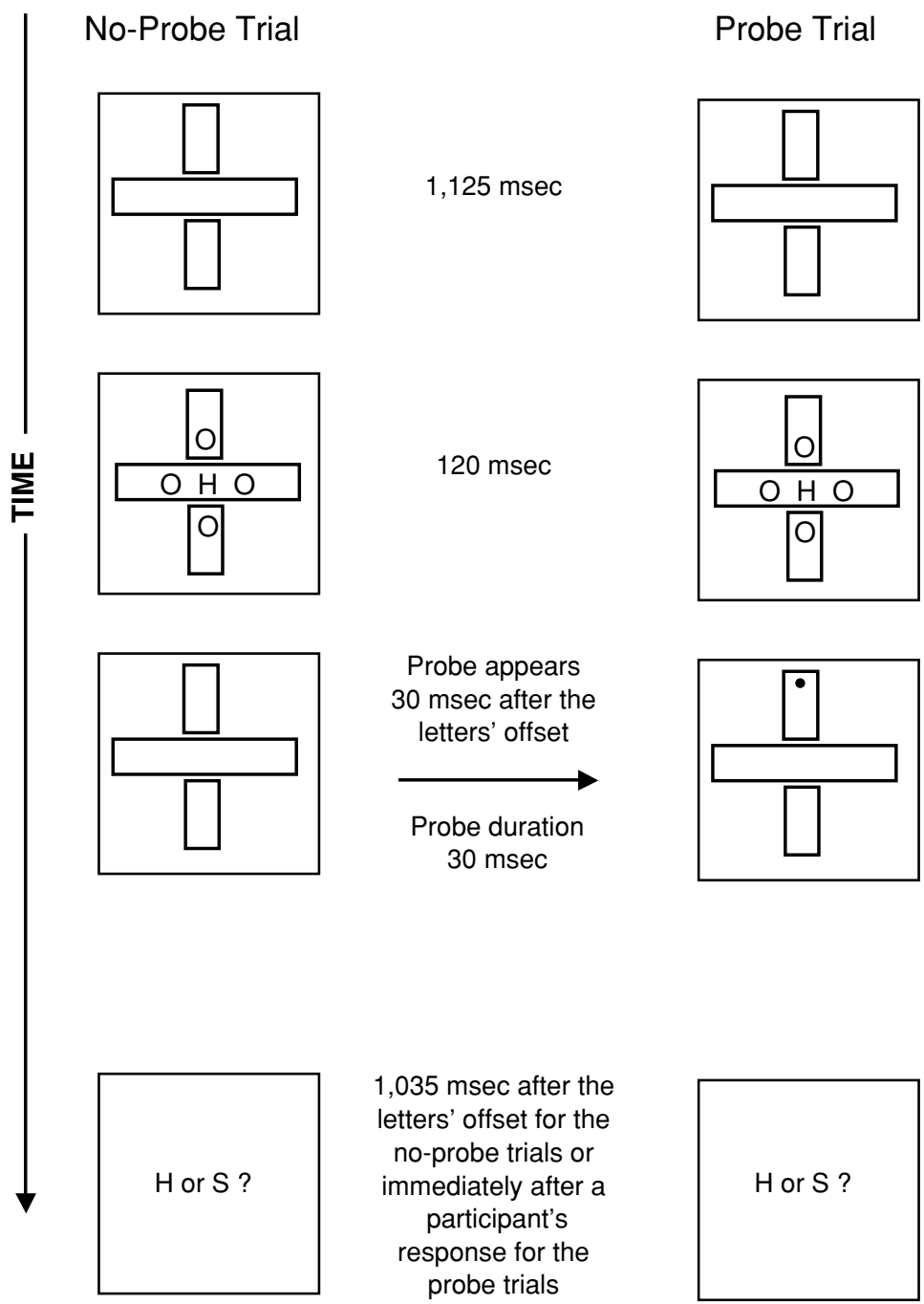

Figure 3. Examples of stimulus displays from Experiment 4. The target was surrounded by four identical flankers, and the probe appeared inside the object boundaries on probe trials. All other aspects of the method were identical to those of Experiment 3.

for the peripheral locations within the rectangles in the different condition (i.e., rectangles without the target), and these response times were virtually identical whether the filler rectangles were present or not (411 vs. $412 \mathrm{msec}$ ). Because the probe technique has been repeatedly demonstrated as a sensitive measure of spatial attention (Cave \& Zimmerman, 1997; Kim \& Cave, 1995, 1999, 2001; Kramer, Weber, \& Watson, 1997), it is unlikely that our results were due to lack of sensitivity. Instead, the most probable explanation is that a narrow focus of spatial attention is maintained even when the filler trials are included. The finding that the two groups showed similar patterns of data across the central and peripheral probes provides converging evidence to the results of Experiment 3, which showed that the object effect observed in Experiment 2 was unlikely to have been the result of a more relaxed attentional focus caused by the inclusion of the filler trials. Taken together, these results suggest that when a stimulus configuration is perceived as being made of individual objects, object effects can arise without a broad extent of attentional focus.

The second question addressed here is whether the object-based attention found in these experiments is actually the result of a selection of the locations within the rectangle containing the target. Table $4 \mathrm{~A}$ shows that responses to locations within the target rectangle (the center and same conditions) are $12 \mathrm{msec}$ faster with the filler trials than without, which is generally consistent with an object-based attention that is mediated by spatial selection. However, there is no hint of a significant effect, and thus it is impossible to attribute these results to objectdriven location selection. It is certainly possible that these results reflect a selection of location-independent object representations. 
Table 4A

Mean Reaction Times (in Milliseconds) and Error Rates (Percentage Incorrect), With Standard Errors, for the Probe Detection Task of Experiment 4

\begin{tabular}{lcclll}
\hline & \multicolumn{2}{c}{ RT } & & \multicolumn{2}{c}{$\%$ Error } \\
\cline { 2 - 3 } \cline { 5 - 6 } & $M$ & $S E$ & & $M$ & $S E$ \\
\hline Whole Group & & & & \\
$\quad$ Center & 452 & 24.1 & & 0.78 & 0.51 \\
$\quad$ Same & 410 & 21.9 & & 0.84 & 0.32 \\
$\quad$ Different & 411 & 20.1 & & 0.4 & 0.26 \\
Mixed Group & & & & \\
$\quad$ Center & 440 & 13.5 & & 0.78 & 0.78 \\
$\quad$ Same & 398 & 11.1 & & 0.78 & 0.51 \\
$\quad$ Different & 412 & 10.9 & & 0.39 & 0.39 \\
\hline
\end{tabular}

Note-Center, central location; Same, peripheral location on the same object; Different, peripheral location on a different object.

Table 4B

Mean Error Rates (Percentage Incorrect), With Standard Errors, for the Letter Discrimination Task of Experiment 4

\begin{tabular}{lccccc}
\hline & \multicolumn{2}{c}{ Whole Group } & & \multicolumn{2}{c}{ Mixed Group } \\
\cline { 2 - 3 } \cline { 5 - 6 } & \% Error & $S E$ & & \% Error & $S E$ \\
\hline With probe & 3.83 & 1.39 & & 2.03 & 0.78 \\
Without probe & 0.34 & .16 & & 0.1 & 0.07 \\
\hline
\end{tabular}

\section{GENERAL DISCUSSION}

\section{Spatial Certainty}

The results from Experiment 2 demonstrate that object organization can affect visual attention even when target and distractor locations are known with complete certainty. Spatial certainty may be necessary to eliminate object-based attention, but spatial certainty alone is not sufficient.

\section{Search Priority}

As described earlier, Shomstein and Yantis (2002) explained their results by positing that object-based attention reflected the priorities with which display items were designated as search targets. In all of the experiments presented here, the target location is known, and there is no need for search. Nonetheless, in these new experiments the object effect still appears. It is difficult to understand how expectations generated by earlier trials about the number of objects to appear could turn a nonsearch task into a search task.

It is also not clear how the search priority account explains results from Harms and Bundesen (1983), Kim and Cave (2001), and Kramer and Jacobson (1991). These experiments all show that grouping affects the allocation of attention despite positional certainty. Shomstein and Yantis (2002) address one part of the Kramer and Jacobson study by proposing that connecting lines can cause target and distractors to be selected together as a single object. They conclude that in their experiments, the distractors were not selected with the target because they were "individuated letters." However, the stimuli in the present experiments were also individuated elements with no connecting lines, as were some of the stimuli used by Kramer and Jacobson, as well as those used by Harms and Bundesen and by Kim and Cave. Taken together, these results suggest that objectbased selection is not simply the result of the priority that different locations receive in visual search.

\section{Top-Down Interpretation of Ambiguous Configurations}

In Experiment 2, the stimuli were exactly like those in Experiment 1, in which there was no object effect. The only difference was the presence of filler stimuli in Experiment 2 that prompted the perception of the rectangles as two separate objects. The results of Experiment 1 suggested that when attention was narrowly focused on the target location, the rest of the configuration would not be processed fully enough for the rectangles to be interpreted as separate objects. However, the results of Experiment 2 showed that even when attention was narrowly focused on the target, an active top-down interpretation of the configuration could cause it to be perceived as multiple objects even without the benefits of diffuse attention, thus producing object-based effects.

In other experiments (Chen \& Cave, 2006), we continued to explore the factors governing the presence of objectbased attention. In those experiments, target locations were known in advance, as in the experiments presented here, but there were two targets instead of one, and they had to be compared against one another. In that study, the presence of object-based attention in one set of trials was affected by the nature of trials in an earlier block, showing once again that for at least some visual tasks, objectbased attention is optional and is governed by top-down processes shaped by experience.

\section{REFERENCES}

AtChley, P., \& Kramer, A. F. (2001). Object and space-based attentional selection in three-dimensional space. Visual Cognition, 8, 1-32.

Avrahami, J. (1999). Objects of attention, objects of perception. Perception \& Psychophysics, 61, 1604-1612.

Cave, K. R., \& Zimmerman, J. M. (1997). Flexibility in spatial attention before and after practice. Psychological Science, 8, 399-403.

CHen, Z. (1998). Switching attention within and between objects: The role of subjective organization. Canadian Journal of Experimental Psychology, 52, 7-16.

CHEN, Z., \& CAVE, K. R. (2006). Object-based attention is affected by time and experience and not by processing load or abrupt onsets. Manuscript in preparation.

Downing, C. J., \& Pinker, S. (1985). The spatial structure of visual attention. In M. I. Posner \& O. S. M. Marin (Eds.), Attention and performance XI (pp. 171-187). Hillsdale, NJ: Erlbaum.

Duncan, J. (1984). Selective attention and the organization of visual information. Journal of Experimental Psychology: General, 113, 501-517.

Egly, R., Driver, J., \& Rafal, R. D. (1994). Shifting visual attention between objects and locations: Evidence from normal and parietal lesion participants. Journal of Experimental Psychology: General, 123, 501-517.

Eriksen, B. A., \& EriKsen, C. W. (1974). Effects of noise letters upon the identification of a target letter in a nonsearch task. Perception \& Psychophysics, 16, 143-149.

Eriksen, C. W., \& Hoffman, J. E. (1974). Selective attention: Noise suppression or signal enhancement? Bulletin of the Psychonomic Society, 4, 587-589.

ERIKSEN, C. W., \& St. James, J. D. (1986). Visual attention within and around the field of focal attention: A zoom lens model. Perception \& Psychophysics, 40, 225-240. 
GARNER, W. R. (1970). The stimulus in information processing. American Psychologist, 25, 350-258.

GARNER, W. R. (1974). The processing of information and structure. Hillsdale, NJ: Erlbaum.

GaRner, W. R., \& Felfoldy, G. L. (1970). Integrality of stimulus dimensions in various types of information processing. Cognitive Psychology, 1, 225-241.

Goldsmith, M., \& Yeari, M. (2003). Modulation of object-based attention by spatial focus under endogenous and exogenous orienting. Journal of Experimental Psychology: Human Perception \& Performance, 29, 897-918.

GotTWALD, R. L., \& GaRNER, W. R. (1975). Filtering and condensation tasks with integral and separable dimensions. Perception \& Psychophysics, 18, 26-28.

Harms, L., \& Bundesen, C. (1983). Color segregation and selective attention in a nonsearch task. Perception \& Psychophysics, 33, 11-19.

Kim, M.-S., \& CAVE, K. R. (1995). Spatial attention in visual search for features and feature conjunctions. Psychological Science, 6, 376-380.

KIM, M.-S., \& CAVE, K. R. (1999). Top-down and bottom-up attentional control: On the nature of interference from a salient distractor. Perception \& Psychophysics, 61, 1009-1023.

KIM, M.-S., \& CAVE, K. R. (2001). Perceptual grouping via spatial selection in a focused-attention task. Vision Research, 41, 611-624.

Kramer, A. F., \& Jacobson, A. (1991). Perceptual organization and focused attention: The role of objects and proximity in visual processing. Perception \& Psychophysics, 50, 267-284.

Kramer, A. F., Weber, T. A., \& Watson, S. E. (1997). Object-based attentional selection - Grouped arrays or spatially invariant representations? Comment on Vecera and Farah (1994). Journal of Experimental Psychology: General, 126, 3-13.

LaBerge, D., \& Brown, V. (1989). Theory of attentional operations in shape identification. Psychological Review, 96, 101-124.

Posner, M. I., SNyder, C. R. R., \& Davidson, B. J. (1980). Attention and the detection of signals. Journal of Experimental Psychology: General, 109, 160-174.

Shomstein, S., \& Yantis, S. (2002). Object-based attention: Sensory modulation or priority setting. Perception \& Psychophysics, 64, 41-51.

Vecera, S. P., \& FARAH, M. J. (1994). Does visual attention select objects or locations? Journal of Experimental Psychology: General, 123, 316-320.

Watson, S. E., \& Kramer, A. F. (1999). Object-based visual selective attention and perceptual organization. Perception \& Psychophysics, 61, 31-49.

YANTIS, S. (1992). Multielement visual tracking: Attention and perceptual organization. Cognitive Psychology, 24, 295-340.

\section{NOTES}

1. Reaction times greater than $1,500 \mathrm{msec}$ were excluded from analysis. Such times accounted for less than $1 \%$ of the total data in all the experiments reported in this paper.

2. We also tested the perception of Egly et al.'s stimuli versus the stimuli used by Shomstein and Yantis. We recruited 16 new participants. Each was shown a single stimulus pattern. A total of four patterns were used: two from the present experiments and two from Egly et al.'s study. Before a participant was shown a pattern, he or she was instructed to describe it with the first thing that came to mind. Interestingly, all of the participants who saw the stimulus patterns from the present experiments described them in ways that suggested a single entity (e.g., "cross," "God," or "Jesus"). In contrast, the responses to the stimulus patterns from Egly et al.'s study were more varied. They included expressions that suggested two different objects (e.g., "two oblongs," "two bricks," "lips," etc.). These results provide converging evidence that the two types of stimulus patterns may differ in a nontrivial way.

3. We thank an anonymous reviewer for making this suggestion.

\section{APPENDIXA}

\section{Display 1}

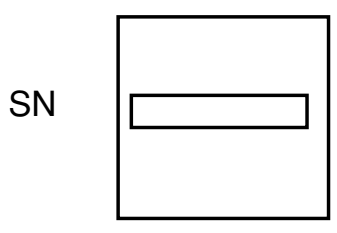

SI
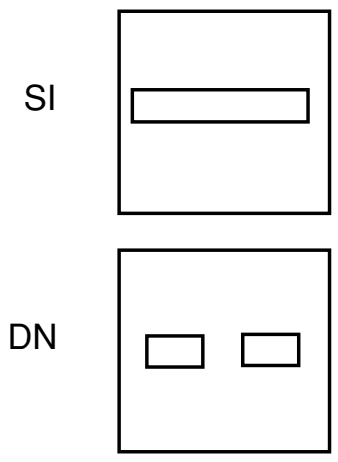

DI

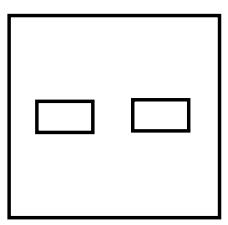

Display 2
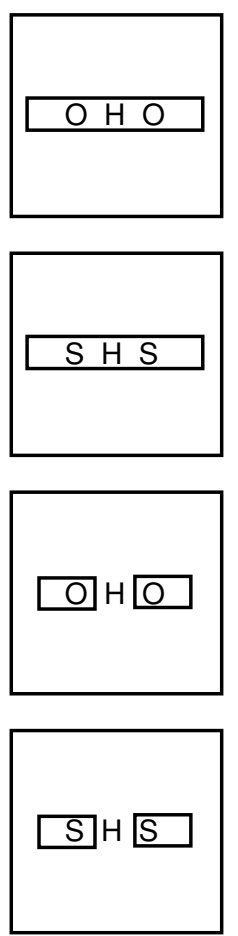

Figure A1. Examples of stimulus displays from the filler trials of Experiment 2. SN is equivalent to the same-neutral condition of the experimental trials; SI, the same-incompatible condition; DN, the different-neutral condition; and DI, the differentincompatible condition. Although the stimuli shown here are all horizontally oriented, on half of the trials they were oriented vertically.

\section{APPENDIX B}

Table B1

Mean Reaction Times (in Milliseconds) and Error Rates (Percentage Incorrect), With Standard Errors, for the Filler Trials of Experiment 2

\begin{tabular}{lrrrrr}
\hline & \multicolumn{2}{c}{ RT } & & \multicolumn{2}{c}{$\%$ Error } \\
\cline { 2 - 3 } \cline { 5 - 6 } & $M$ & $S E$ & & $M$ & $S E$ \\
\hline Same Object & & & & \\
I & 460 & 13.3 & & 5.8 & 0.99 \\
N & 427 & 12.7 & 2.2 & 0.35 \\
I - N & 33 & 3.1 & 3.7 & 0.82 \\
Different Object & & & & \\
I & 433 & 11.9 & & 4.3 & 1.16 \\
N & 423 & 11.9 & 2.7 & 0.57 \\
I - N & 10 & 3.4 & 1.6 & 1.06 \\
\hline
\end{tabular}

Note-I, incompatible; N, neutral. 


\section{APPENDIX C}

Table C1

Mean Reaction Times (in Milliseconds) and Error Rates (Percentage Incorrect), With Standard Errors, for the Probe Detection Task of the Filler Trials of Experiment 3

\begin{tabular}{lccccc}
\hline & \multicolumn{2}{c}{$\mathrm{RT}$} & & \multicolumn{2}{c}{$\%$ Error } \\
\cline { 2 - 3 } \cline { 5 - 6 } & $M$ & $S E$ & & $M$ & $S E$ \\
\hline Center & 480 & 29.1 & & 2.34 & 1.64 \\
Peripheral & 450 & 33.4 & & 1.37 & 0.55 \\
\hline
\end{tabular}

Table C2

Mean Error Rates (Percentage Incorrect), With Standard Errors, for the Letter Discrimination Task of the Filler Trials of Experiment 3

\begin{tabular}{lcc}
\multicolumn{3}{c}{ Filler Trials of Experiment 3 } \\
\hline \%ith probe & 7.69 & $S E$ \\
\hline Without probe & 0.85 & 0.43 \\
\hline
\end{tabular}

\section{APPENDIX D}

Table D1

Mean Reaction Times (in Milliseconds) and Error Rates (Percentage Incorrect), With Standard Errors, for the Probe Detection Task of the Filler Trials of Experiment 4

\begin{tabular}{lccccc}
\hline & \multicolumn{2}{c}{ RT } & & \multicolumn{2}{c}{$\%$ Error } \\
\cline { 2 - 3 } \cline { 5 - 6 } & $M$ & $S E$ & & $M$ & $S E$ \\
\hline Center & 425 & 7.4 & 0 & 0 \\
Same & 407 & 9.7 & 0 & 0 \\
Different & 401 & 9.3 & 0 & 0 \\
Outside & 407 & 10.9 & 3.1 & 0.84 \\
\hline
\end{tabular}

Note-Center, central location; Same, peripheral location on the same object; Different, peripheral location on a different object; Outside, outside the object boundaries.

Table D2

Mean Error Rates (Percentage Incorrect), With Standard Errors, for the Letter Discrimination Task of the Filler Trials of Experiment 4

\begin{tabular}{lcc}
\hline & \multicolumn{2}{c}{$\%$ Error } \\
\cline { 2 - 3 } & $M$ & $S E$ \\
\hline With probe & 7.98 & 0.74 \\
Without probe & 0.26 & 0.13 \\
\hline
\end{tabular}

(Manuscript received January 14, 2004;

revision accepted for publication September 30, 2005.) 